\title{
Chronic Obstructive Pulmonary Disease (COPD) and Its Association with Lung Cancer: Molecular Mechanism and Therapeutic Targets
}

\author{
Shanmugam G, Rakshit S and Sarkar K* \\ Department of Biotechnology, SRM Institute of Science \\ and Technology, Kattankulathur, Tamil Nadu 603203, \\ India \\ *Corresponding author: Koustav Sarkar, Department \\ of Biotechnology, SRM Institute of Science and \\ Technology, Kattankulathur, Tamil Nadu 603203, India
}

Received: May 19, 2021; Accepted: J une 15, 2021; Published: J une 22, 2021

\begin{abstract}
Chronic Obstructive Pulmonary Disease (COPD) and Lung cancer are the major reasons for lung disease-related mortality worldwide. Chronic inflammation is a key attribute of COPD and a potential driver of lung carcinogenesis. Among various environmental risk factors, cigarette smoke plays a crucial role in the development and progression of COPD and lung cancer. Several epidemiological studies show that COPD patients are at a greater risk of developing lung cancer independently of cigarette smoking which suggests the role of genetic predisposition in the disease development. Uncovering the mechanistic link between these two diseases is hampered due to their heterogeneous nature: each is characterized by several sub-phenotypes of diseases. This review focuses on the nature of the link between the two diseases and specific mechanisms that occur in both COPD and lung cancer, some of the therapeutic targets which are currently employed, and the role of gene-editing technology to combat these debilitating lung-inflammatory disorders.
\end{abstract}

Keywords: Chronic inflammation; Lung cancer; Chronic obstructive pulmonary disease; Genetic predisposition; Therapeutic targets

\section{Abbreviations}

COPD: Chronic Obstructive Pulmonary Disease; SCLC: Small Cell Lung Cancer; NSCLC: Non-Small Cell Lung Cancer; GWAS: Genome-Wide Association Studies; SNP: Single Nucleotide Polymorphism; nAChRs: nicotinic Acetylcholine Receptors; CXCL14: C-X-C Motif Chemokine Ligand 14; EWAS: EpigenomeWide Association Study; DNMT1: DNA Methyltransferase 1; Sp1: Specificity Protein 1; NNK: Nicotine-Derived Nitrosamine Ketone; HAT: Histone Acetyltransferase; HDAC: Histone Deacetylase; HMT: Histone Methyltransferase; HDM: Histone Demethylase; PI3K: Phosphoinositide-3 Kinase; LSD1: Lysine-Specific Demethylase 1; TNF-a: Tumor Necrosis Factor a ; ROS: Reactive Oxygen Species; NRF2: Nuclear Factor Erythroid 2-Related Factor 2; MAVS: Mitochondrial Antiviral Signaling Protein; Mfn-2: Mitochondrial Fusion Protein; HIF: Hypoxia-Inducible Factor; SAA: Serum Amyloid A.

\section{Introduction}

COPD is a chronic inflammatory lung disease that causes airflow obstruction in the lungs. COPD prevalence, mortality, and morbidity vary across countries but it is substantial and increasing. Approximately 251 million people are suffering globally. COPD affects $8-10 \%$ of adult populations and $15-20 \%$ of smokers in developed and developing countries. The World Health Organisation predicts COPD will become the third leading cause of death globally by 2030 [1-3].

Lung cancer is the most common malignancy and the leading cause of cancer death worldwide. Lung cancer is histologically classified into two types; Small Cell Lung Cancer (SCLC) and Non-
Small Cell Lung Cancer (NSCLC). Among these two, NSCLC accounts for $80 \%$ of lung cancer cases. Lung cancer is the most frequently diagnosed cancer (11.6\%) followed by breast and colorectal cancer. It is the leading cause of death at 1.74 million worldwide. Worldwide lung cancer incidents are expected to increase by $38 \%$ to 2.89 million by 2030 . The five-year cancer survival rate for all types of lung cancer is $19 \%$ which is lower than other cancers including colon, breast, and prostate [4]. Cigarette smoke exposure is a shared environmental risk factor in COPD and lung cancer [5]. However, non-smokers also develop these diseases which highlights the importance of genetic predisposition [6].

A nested case-control study of the genetic epidemiology of COPD (COPDGene) showed that COPD severity can be a predictive tool for lung cancer [7]. It is already shown that COPD increases the risk of lung cancer up to 4 to 5 fold. COPD is known to drive lung tumorigenesis by increasing oxidative stress, repression of DNA repair mechanisms, increased expression of pro-inflammatory cytokines, and cellular proliferation. Smoking-associated COPD is aligned with the development of NSCLC and SCLC [8].

This review will mainly focus on the current understanding of common pathogenic pathways contributing to COPD and lung cancer, and how environmental factors like cigarette smoke influences the expression of genes via epigenetic modification and the therapeutic strategies to target these diseases.

\section{Molecular Aspects Linking COPD and Lung Cancer}

Pathogenesis of Chronic Obstructive Pulmonary Disease and lung cancer are believed to be linked. Inhalation of more than 6000 


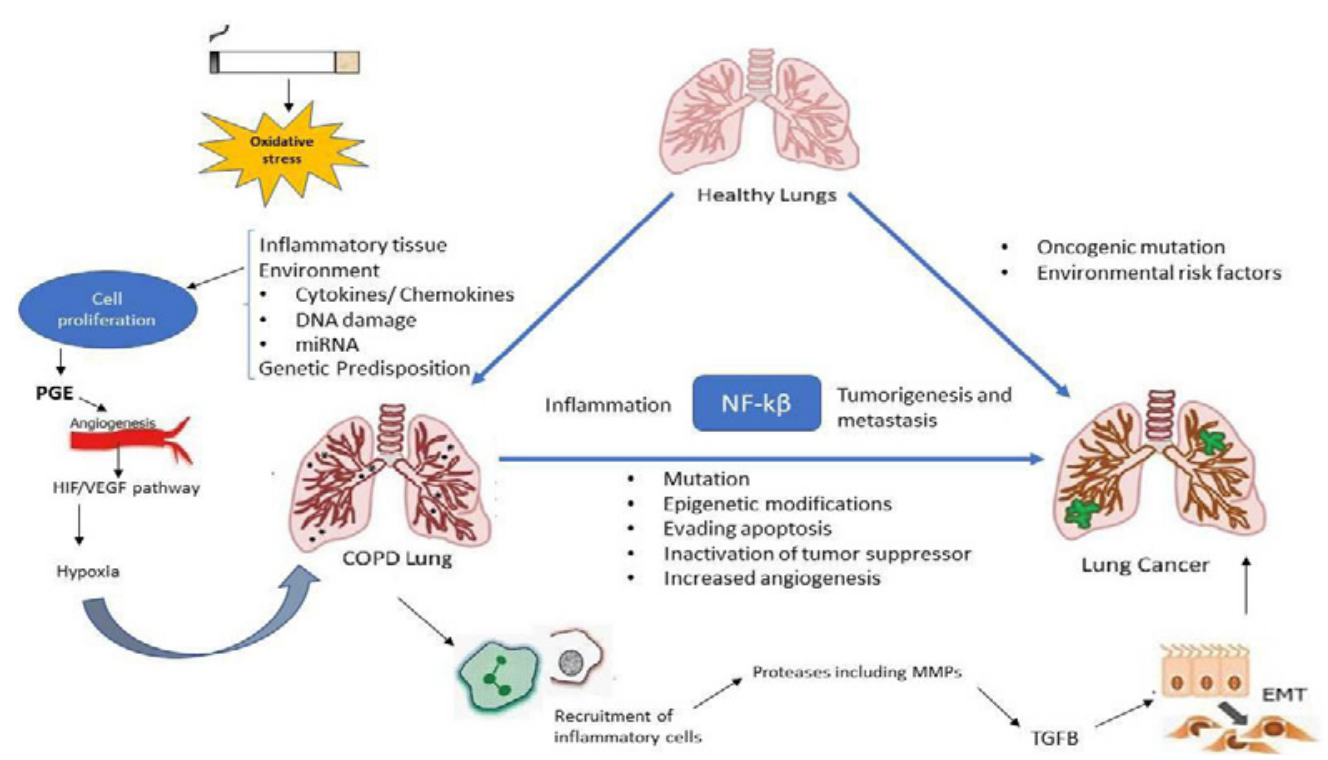

Figure 1: Linkage of lung cancer and COPD owing to their common exogenous and endogenous factors. Both share similar pathogenic mechanism which includes $\mathrm{NF}-\mathrm{kB}$, oxidative stress, hypoxia and angiogenesis.

compounds present in cigarette smoke becomes the common risk factor for both the diseases. However, only a fraction of long-term smokers develop COPD and/or lung cancer. These observations, together with the familial aggregation of indicate the contribution of genetic components to these disorders [9]. Various mechanisms like genetic susceptibility, epigenetic alteration, DNA damage, and repair, down regulation of specific microRNA, expression of proinflammatory genes, and immune responses have been proposed to explain the association between COPD and lung cancer [10] (Figure 1).

\section{Genetic Predisposition in COPD and Lung Cancer}

A large amount of genetic variation and environmental risk factors greatly influence the pathogenicity of COPD and lung cancer. Genome-wide Association Studies (GWAS), Whole Genome sequencing, transcriptome, and whole gene methylation are useful in studying the genetic variation of COPD and lung cancer [11-13].

\section{SNP's in COPD and Lung Cancer}

The Single Nucleotide Polymorphism (SNPs) is the most common genetic variation which involves the substitution of a single nucleotide at a specific position. Pathogenic genes are believed to have high frequencies of SNP variations. SNP variations of genes like SNPsrs660652 and rs12910984 in the nicotinic Acetylcholine Receptors (nAChRs) family are related to COPD and lung cancer [14]. Variants in CHRNA5-HRNA3-CHRNB4 locus are strongly associated with smoking. Among SNP variations in nAChRs, rs16969968 was confirmed to be associated with lung cancer and COPD and hence proposed as the best potential target gene in COPD-Lung cancer transformation [15].

In addition to this SNP variation of inflammatory genes play a crucial role. rs1800469 in the promoter region of the TGFB1, rs139092247, and rs2568494 in IREB2, rs117916638 in EGLN2, position-308 of TNF-alpha were associated with cigarette smokers with COPD and lung cancer [16-19].

\section{Differentially Expressed Genes in COPD and Lung Cancer}

The copy number variation and expression of genes are equally important while exploring the association of COPD with lung cancer. CHRNA7 is up-regulated and proposed as a genetic biomarker of the co-existence of both diseases [20]. HER2 was overexpressed in patients with COPD and lung cancer but not in lung cancer patients. Hence it might be involved in the regulation of lung epithelial cell transformation from chronic inflammation to lung tumorigenesis [21]. The C-X-C motif chemokine ligand 14 (CXCL14) is involved in the transformation from smoking to lung cancer through upregulated expression. The expression of CXCL14 is high in the airway epithelium of healthy smokers, and it is furthermore in smokers with COPD, and most up-regulated in smokers with COPD and lung ADC [22]. The expression of a COPD-associated CXCL14-correlating cluster of genes was associated with a poor survival rate of patients with COPD and lung ADC. Reports by Wang et al., after analyzing gene expression profiles showed that some candidate genes are up or down-regulated in smokers with COPD or lung cancer and COPD [23].

\section{Epigenetic Dysregulation in COPD and Lung Cancer}

Epigenetics is defined as the study of heritable or acquired stable changes in gene expression that occur without variation in the DNA sequence. DNA methylation, post-translational histone modifications (including histone acetylation, methylation, phosphorylation, ubiquitination, and sumoylation) are the three main epigenetic marks. Evidence has increased that epigenetic mechanisms influence gene expression in chronic lung diseases like Chronic Obstructive Pulmonary Disease (COPD). Epigenetic silencing of genes through promoter methylation of cytosines and histone modifications is now 
recognized as a major and normal event affecting the expression of hundreds of genes during initiation and clonal development of lung cancer.

\section{DNA Methylation and CpG Island in COPD \& Lung Cancer}

Epigenome-Wide Association Study (EWAS) is carried out to study the link between gene methylation in COPD and lung cancer. Repression of CCDC37 and MAP1B genes is significantly associated with both COPD and lung cancer [24] Immune-modulatory genes are hypomethylated to a greater extent in COPD and Lung cancer patients [25].

Clinical data from lung cancer patients have shown that DNA Methyltransferase 1 (DNMT1) is overexpressed which catalyzes methylation of DNA in CpG islands leads to p53 mutation and increased expression of specificity protein 1 (Sp1) [26-28]. Nicotinederived nitrosamine ketone (NNK) is also playing a major role in epigenetic alterations like hypermethylation of promoters of multiple tumor suppressor genes by induced activation of DNMT1 [29]. Identification of $\mathrm{CpG}$ methylation events in COPD as a precursor event in lung cancer will be of valuable clinical significance. Examples of such candidate genes that are hypermethylated in both diseases are IL-12R $\beta 2$, Wif-1, P16, GATA4, KEAP1, and ECSOD [30].

\section{Histone Modification in COPD and Lung Cancer}

Histone modification is a cellular process where histone tails are modified by nonhistone chromatin-associated proteins called chromatin-modifying enzymes. Various post-translational modifications in histones play a crucial role in genomic functions which in turn alter the structural property of chromatin [31]. Based on their function chromatin modification enzymes are classified, for example, acetylation by Histone Acetyltransferases (HATs), deacetylation by Histone Deacetylases (HDACs), methylation by Histone Methyltransferases (HMTs), and demethylation by Histone Demethylases (HDMs).

The resulting Post-translational modification will promote the activation or repression of chromatin-mediated gene expression for inflammatory mediators, apoptosis, senescence, growth factors, and tumor suppressor genes involved in COPD and lung cancer $[32,33]$. The activities of these enzymes are greatly influenced by environmental cues like prolonged exposure to irritants or particulate matter most often involves cigarette smoke which occurs in patients with COPD and lung cancer [34].

\section{Cigarette Smoke Mediated Epigenetic Modification in COPD \& Lung Cancer}

Cigarette smoke is one of the most important risk factor in the development of COPD emphysema and lung cancer. It contains more than 1014 oxidants and about 4700 reactive chemical compounds. Cigarette smoke challenges the lung through the generation and release of ROS and by activating various redox-sensitive transcription factors, which ultimately leads to increased expression of proinflammatory cytokines and chemokines. The next major impact of cigarette smoke is chromatin modification and epigenetic changes by causing post-translational modifications to HDAC2 and SIRT1 which ultimately results in chromatin remodelling [35].

HDAC2 is an important redox-sensitive protein and it prone to alteration by oxidants and free radicals which leads to decreased activity of this deacetylase enzyme. It has been reported that a significant reduction in the activity of HDAC2 in the lungs of smokers and patients with COPD compared to healthy non-smokers [36-38]. Several in vitro experiments showed that in response to cigarette smoke there is a decreased level of HDAC2 in human airway and bronchial epithelial cells and lungs of mice. This study helps to understand that HDAC2 could be modified post-translationally by aldehydes and protein nitration present in cigarette smoke [39]. HDAC2 deacetylase activity is decreased in monocytes, epithelial cells, and rodent lungs due to nitration of tyrosine residues on HDAC2 by nitric oxide (NO) [40]. Moreover, the level of nitrated HDAC2 is significantly increased in alveolar macrophages from patients with COPD [41]. The activity of HDAC2 has been drastically reduced by oxidative stress by proteasomal degradation. A basal level of phosphorylation is required for HDAC2 deacetylase activity [42]. Hyperphosphorylation of HDAC2 results in the dissociation of co-repressor complex and enhanced ubiquitination and finally degradation. Cigarette smoke induces increased phosphorylation of HDAC2 by a kinase-dependent mechanism $[43,44]$. Regulation of HDAC2 activity by casein kinase 2 (CK2- downstream of phosphatidylinositol 3-kinase (PI3K)) is validated by Marwick and colleagues where they showed that stability and lack of phosphorylation of HDAC2 in phosphoinositide-3 kinase (PI3K) $\delta$ knockout mice in response to cigarette smoke [45].

SIRT1 is another class of histone deacetylase enzyme that deacetylase both histone and non-histone proteins including transcription factors even other signaling molecules. Cigarette smoke exposure has drastically reduced the level of SIRT1 in the lungs of smokers and patients with COPD. SIRT1 undergo post-translational modification and there is increased nitration and carbonylation of SIRT1 were observed in the lungs of smokers on exposure to cigarette smoke and patients with COPD [46]. Disruption of SIRT1-RelA/p65 complex occurs once there is a degradation of SIRT1 after PTM which ultimately results in the release of high pro-inflammatory mediators [47] this high expression of pro-inflammatory genes leads to chronic inflammation which is the initial stage in the pathogenesis of COPD \& lung cancer.

Thus Cigarette smoke greatly influences the development and progression of COPD and lung cancer by increasing the oxidative stress and the activation of various redox-sensitive transcription factors and chromatin remodeling by alteration in the redox- sensitive deacetylases.

Acetylation of histone $\mathrm{H} 3$ in macrophages and lungs of humans and rodents were observed in response to cigarette smoke, this work elucidates the role of histone acetylation in chromatin remodeling and subsequent activation of an inflammatory response in lungs of COPD patients [48-51].

Histone Methyl Transferases will affect global methylation and are deregulated in several types of cancers. Gene activation and repression depend on the methylation level of histones. For example, when $\mathrm{H} 3 \mathrm{~K} 4, \mathrm{H} 3 \mathrm{~K} 36$, and $\mathrm{H} 3 \mathrm{~K} 79$ are methylated it linked to gene activation, whereas methylation at $\mathrm{H} 3 \mathrm{~K} 9, \mathrm{H} 3 \mathrm{~K} 27$, and $\mathrm{H} 3 \mathrm{~K} 20$ leads to gene repression [52] Histone Demethylases (HDMs) are classified 
into two types, such as lysine-specific demethylase 1 (LSD1) and Jumonji C (JmjC) domain family proteins which are involved in the regulation of gene expression [53]. HDM expression is dysregulated during tumor initiation and progression. It has been observed that there is an alteration in the expression level of JMJD1A, JMJD2B \& JARID1A during hypoxic conditions in the lungs of patients with COPD [54-56].

\section{Role of Inflammation in COPD \& Lung Cancer}

Inflammation is a biological process in which our cells recruit a diverse set of immune cells to fight against pathogens. The small airways of COPD patients are inflamed and there is an alteration in inflammatory cytokine level. Chronic inflammation is due to the activation and recruitment of macrophages, CD4+, and CD8+ T cells, dendritic cells, B cells, and neutrophils. Some studies showed that the degree of inflammation directly correlates with COPD severity and both COPD \& Lung cancer are associated with an inflammatory environment.

Reports showed that upregulation of Tumor necrosis factor a (TNF- $\alpha$ ) in COPD induces an immune suppressive effect within the tumor microenvironment thereby promoting lung tumorigenesis [57]. A high level of TNF- $\alpha$ is observed in tumor samples from lung cancer patients [58]. Bioinformatic analysis also highlighted that innate defense, lymphocyte trafficking, and dendritic cell pathways are enriched in COPD associated lung cancer. Some of the most important inflammatory mediators and intracellular signaling pathways involved in the pathogenesis of lung cancer and COPD are as follows.

\section{Oxidative Stress and Mitochondrial Damage in COPD and Lung Cancer}

Several mechanisms have been proposed to account for the association between COPD and lung cancer. Among these, oxidative stress and chronic inflammation are playing a crucial role in the process of carcinogenesis. Mitochondrial dysfunction is the key player of these two conditions which promotes tumorigenesis in COPD patients. Excessive Reactive Oxygen Species (ROS) exposure to airways leads to an increase in oxidative stress among COPD patients compared with non-COPD smokers. Mitochondrial ROS is increased in the airways of patients with COPD which is the effect of mitochondrial damage [59]. Impairment of antioxidant NRF2 (nuclear factor erythroid 2-related factor 2) and various pathways involved in oxidant and antioxidant responses are differentially expressed in COPD patients $[60,61]$. Oxidative stress-related proteins like catalase and thioredoxin are differentially expressed in both COPD and COPD-associated NSCLC compared with nonsmokers [62]. Induction of cellular senescence is mediated by increased oxidative stress which aids in the process of carcinogenesis. Mitochondria are the main source of ROS, from the oxidative phosphorylation system. Also, it acts as a sensor for inflammatory damage signals and it is the site of initiation of inflammatory response. Mitochondrial Antiviral Signaling protein (MAVS) initiates the NLRP3 inflammasome, a multimolecular complex .Change in the conformation of this inflammasome results in the activation of caspase- 1 and hence induces the expression of pro-inflammatory cytokines, like Interleukin (IL)-1 $\beta$ and IL-18 [63].
Cigarette smoke affects the structure and function of mitochondria thus leads to increased expression of the mitochondrial fusion protein, Mfn-2, in alveolar epithelial cells [64]. Work done by Lou et al., shows that the expression of Mfn- 2 is high in lung adenocarcinoma when compared to adjacent normal lung tissue [65]. Damaged mitochondria undergo uncoupling and release of increased ROS. Mitophagy is activated to clear the damaged mitochondria and if mitophagy is impaired then there will be mitochondrial DNA accumulation in the cytoplasm.

\section{Immune Defects in COPD and Lung Cancer}

Reports are there to show that adaptive immune response also involves in the pathogenesis of COPD because mature lymphoid follicles with a germinal center and separated $\mathrm{T}$ and $\mathrm{B}$ cells zone occur in the lungs of COPD patients and it associates with the severity of COPD [66]. There is also marked alteration of the innate immune system in COPD. COPD patients have intense immune dysregulation which leads to enhanced activity of regulatory T cells, $\mathrm{CD} 4^{+}, \mathrm{PD}-1+$ exhausted effector T cells, and myeloid-derived suppressor cells. Mast cell activation is also aberrant in the lungs of COPD and lung cancer patients [67]. In addition to that, natural killer $\mathrm{T}$ cells, a cellular interface between innate and adaptive immunity also deregulated in COPD [68]. There are some specific mediators like Serum Amyloid A (SAA) implicate the links between COPD and Lung cancer. SAA is highly expressed in COPD and it helps the production of tumors [69]. In response to cigarette smoke, alveolar macrophages (AM) in COPD patients have an impaired phagocytic capacity which is associated with the deregulated release of extracellular sphingosine-1-phosphate (S1P) released from airway epithelial cells [70]. Mitochondrial dysfunction occurs in COPD and lung cancer results in impairment of the HMG-CoA reductase pathway which is involved in the production of cholesterol, and all steroid hormones. The defective HMG-CoA pathway affects innate immune response which might link COPD with an increased incidence of lung cancer [71].

\section{NF-кB Pathway in COPD \& Lung Cancer}

The most important intracellular signaling pathway involves in the pathogenesis of COPD and lung cancer is NF- $\kappa \mathrm{B}$. NF- $\kappa \mathrm{B}$ is a critical pro-inflammatory transcription factor and it is well known that both canonical and non-canonical NF- $\mathrm{kB}$ pathways induce increased expression of pro-inflammatory mediators leading to chronic inflammation in the lung. Several genes including cytokines, adhesion molecules, angiogenic factors, anti-apoptotic factors, and Matrix Metalloproteinases (MMPs) that all have shown to be associated with tumor progression and metastases are regulated by NF- $\kappa B$. STAT 3 and NF- $\kappa B$ activation are key players in the development of lung cancer from COPD. NF- $\kappa B$ suppresses the expression of $\mathrm{p} 53$ thereby stimulates proliferation and inhibit cell death [72]. The pro-inflammatory environment which consists of activated macrophages and regulatory $\mathrm{T}$ cells depends on NF- $\mathrm{kB}$ and links COPD with lung cancer [73].

\section{Gene Editing as a New Therapeutic Target for Lung Cancer \& COPD}

Respiratory diseases ranging from relatively mild to severe conditions such as COPD, Asthma, and lung cancer are influenced by environmental conditions as well as genetic or epigenetic changes as 
their root cause. Hence there is a need for novel tools that manipulate the function of endogenous genes. Gene Editing technology offers tools that allow us to investigate the role of a particular gene in the physiology and pathophysiology of the respiratory system. Epigenetic editing helps to rewrite the epigenetic marks at the defined locus of our gene of interest. Zinc Finger Nucleases (ZFN) and Transcription Activator-Like Effector Nucleases (TALEN) are widely used DNA targeting system until the introduction of the much more straightforward editing platform CRISPR/Cas9. A system of Clustered Regularly Interspaced Short Palindromic Repeats (CRISPR) and CRISPR-associated (Cas) proteins, is now taking the research field to new heights by providing new insights about the underlying mechanism of the diseases.

\section{Application of CRISPR/ Cas9 in Lung Cancer Modelling, Tumorigenesis}

CRISPR/Cas 9 mediated gene editing has accelerated the progress of lung cancer research in any direction by assisting in the identification of target genes, drug-resistance genes, and aids in constructing animal tumor models. One example of this is the deletion of gene 33 which leads to the reduction of cell proliferation and also increases cell migration. Due to this deletion, there is an upregulation of UCHL1 (deubiquitination enzyme) which is associated with the early stages of lung epithelial cell transformation and tumorigenesis [74]. Manguso et al. showed that deletion of PTPN2 improves the efficiency of immunotherapy by enhancing interferon mediated effect on growth suppression and antigen presentation [75]. Inhibition of miR584-ROCK1 pathway by CRISPR Cas9 FLI1 gene knockout results in cessation of tumor progression [76]. Identification of drug resistance genes is done by Wang et al., They have shown that CRISPR Cas9 based knockout of the receptor UPAR (Urokinase plasminogen activator receptor) results in a decrease in resistance of lung cancer cells towards drugs like 5-FU, Doxorubicin, cisplatin, and docetaxel. This knockout also resulted in inhibition of cancer cell proliferation, invasion, and migration [77].

\section{Clinical Trials using CRISPR/Cas9 Genome Editing}

Even though being a potential genome editing tool, very few CRISPR/Cas9 projects got approved to conduct human clinical trials. One study involves direct administration of the CRISPR/Cas9 system into the human body after editing the immune checkpoint genes to improve the efficacy of T cell therapy in advanced NSCLC patients. It mainly aims to evaluate the safety of $\mathrm{PD}-1$ knockout engineered $\mathrm{T}$ cells and the results from a first-in-human phase I clinical trial (ClinicalTrials.gov NCT02793856) showed that it is safe and feasible [78].

\section{Application of CRISPR/Cas9 in COPD}

Till now very limited reports are available regarding the application of gene editing tools in COPD. Hypersecretion of airway mucus is one of the main causes of the pathogenesis of COPD. SPDEF (SAMpointed domain-containing Ets-like factor) is a master regulator of mucus production. Song et al. showed that targeted silencing of SPDEF using ZFPs and CRISPR/Cas9 has successfully reduced the mucus-related gene expression which might be a new therapeutic approach for patients with excessive mucus secretion [79].
Epigenetic editing of gene expression in the lung is complicated due to the difficulties in delivering the epigenetic editor. Several barriers like Physical and immune barriers of lung hamper successful delivery to the lung. Excessive mucus production and inflammation will further worsen the situation. To tackle these problems, several gene transfer systems were developed which employ viral vectors like Retroviral, Adenoviral. Thus Gene editing based technologies like CRISPR systems shows promising therapeutic possibilities for lung and respiratory tract diseases.

\section{Therapeutic Targets in COPD and Lung Cancer}

COPD and lung cancer are debilitating lung disorders whose underlying molecular and cellular mechanisms are not completely understood. Many patients fail to respond to broad-spectrum antiinflammatory molecules like corticosteroids. Bronchodilators have been advised to the patient as a part of the management of the disease; however, it fails to target chronic inflammatory response which is a key player in both disorders. This implies the need for finding a novel therapeutic target for COPD and lung cancer.

Several studies support the role of chemokines and their receptors including chemokine CXCR2 and CXCR3 with smallmolecule receptor antagonist (SB332235, NBI74330) [89]. This antiinflammatory therapy will reduce the inflammatory profile in COPD, which is unaffected by existing pharmacotherapy.

Inhibitors that target the core component of apoptotic signaling are also at an early stage of development. Selective inhibitors of caspases-3, 7, and 9 are carried out by IAPS- Inhibitors of apoptosis proteins. Enabling apoptosis to take place could be a potential treatment of malignancy and thus several agents that target IAPs are gaining more interest [90].

Antioxidant therapy is one of the emerging tools which target oxidative stress with antioxidants or boosting the endogenous level of antioxidants. Thiol molecules (glutathione and mucolytic drugs, such as $\mathrm{N}$-acetyl-L-cysteine and $\mathrm{N}$-acystelyn), dietary polyphenols (curcumin, resveratrol, green tea, catechins/quercetin), erdosteine, which have been reported to control oxidative stress by inhibiting NF-КB activation and maintaining the balanced redox state of the cell. Thus administration of multiple antioxidants will be effective in the treatment of lung diseases [91].

Proteomic profiling studies help to identify the dysregulated pathways in small cell lung cancer which leads to the development of therapies that specifically target those pathways. There is emerging evidence shows that the MAPK pathway contributes to several COPD-associated phenotypes. The classical MAP kinase can be inhibited at several points. Inhibition at the membrane blocks receptor dimerization. Inhibitors of Receptor Tyrosine Kinase exist, such as EGFR inhibitors (AG1478, ZD1839). ZD1839 has been tried in a mouse model and it has been shown to block lung injury. These drugs have shown promising results in clinical trials but the adverse effects are an issue. One of the promising compounds is CI-1040. CI1040 (PD 184352), a specific small-molecule inhibitor of MEK1/2 is well tolerated in humans and has shown efficacy as an anti-tumor agent [92]. 


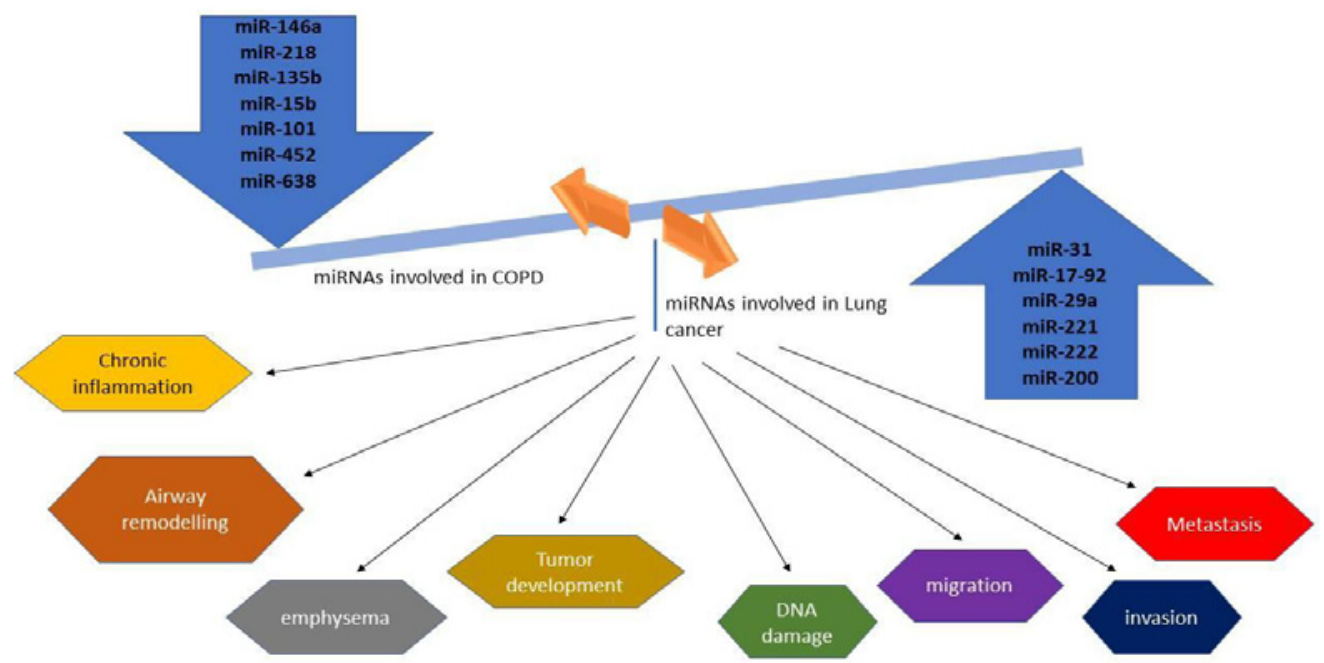

Figure 2: MicroRNA involved in the pathogenesis of Lung cancer and COPD. Dysregulation of these miRNA aids in the development of both disorders.

\section{miRNA as a Feature Therapeutic Target}

MicroRNAs (miRNAs) are small, endogenous non-coding RNAs that regulate gene expression. miRNA bind to its target messenger RNAs in a sequence-specific manner, which leads to direct inhibition of protein synthesis or degradation of the transcript. Several miRNAs are known to regulate genes involving in the process of the developmental and physiological process including cellular proliferation, apoptosis, and differentiation, innate and adaptive immune responses (Figure 2). For instance, miR-146a-5p has been found to be a regulator of inflammation [80]. miR-146a-5p expression is impaired in fibroblast of patients with COPD, which release the break on the IL-1R/NF- $\kappa B$ pathway and contribute to abnormal inflammation. Treating fibroblast with miR-146a-5pmimic results in a reduced level of IL-8. This confirms the anti-inflammatory role of miR-146a-5p [81]. In vitro and in vivo studies have shown the role of miRNA in the pathogenesis of COPD. Recent in vivo and gene set enrichment analysis explained the role of miR-218-5P in cigarette smoke-induced inflammatory response as it was downregulated in the epithelium of COPD patients [82].

Numerous studies have documented the role of miRNA in every process of carcinogenesis of lung cancer including tumor development, metastasis, and drug resistance. Works done by Cho et al. demonstrated that miR-145 restoration inhibits cancer cell growth in EGFR mutant lung cancer cells thus it may serve as a therapeutic target [83]. Identification of oncogenic miRNA (oncomir) like miR-31 helps to repress cancer cell growth and tumorigenicity by increasing the expression of tumor-suppressive genes [84]. miR17-92 has found to regulate the generation of DNA damage in RBinactivated lung cancer cells [85]. Some miRNAs have been shown to target genes related to drug sensitivity that helps to reverse the drug resistance of cancer cells. miR-134 greatly increases the sensitivity to anti-cancer drugs like cisplatin, etoposide, and doxorubicin [86]. Mir-181b expression sensitizes multidrug-resistant lung cancer cells to cisplatin-induce apoptosis by reducing the level of BCL2 protein [87]. Silencing of miR-10b with antagomirs significantly increases the level of Hoxd10 and suppresses lung metastasis in mice bearing high metastatic cells. MiR-10b antagomir is tolerated by normal animals and it is believed to be a potential candidate for the development of new anti-metastasis drugs [88].

Hence miRNAs can be a promising candidate for the new generation therapeutics. The main challenge for this miRNA therapeutics is delivery, stability, and safety. Recent research focuses on developing a stable miRNA delivery vector to appropriate cells within a tissue or organ.

\section{Conclusion}

Both COPD and lung cancer is the most common cause of mortality worldwide and shares key factors including cigarette smoke and harmful gas exposure. Not all smokers develop COPD or lung cancer, indicating the complex interplay between genetics, epigenetics, and environmental factors. Unravelling the precise mechanisms behind the increased incidence of lung cancer among COPD patients are not completely understood due to the heterogeneity of these disorders. However, there are accumulating pieces of evidence which show some of the association between these two diseases. Several mechanisms have been proposed to account for the association between COPD and lung cancer. Among these, oxidative stress and chronic inflammation are the most studied drivers of carcinogenesis. Mitochondrial dysfunction is the key player of these two conditions which promotes tumorigenesis in COPD patients. SNP variations of genes in the nicotinic acetylcholine receptors (nAChRs) family are related to COD and lung cancer. Epigenome-Wide Association Study (EWAS) showed that Repression of CCDC37 and MAP1B genes is significantly associated with both COPD and lung cancer and Immune-modulatory genes are hypo methylated to a greater extent in COPD and Lung cancer patients. Further reports showed that upregulation of Tumor necrosis factor $\alpha$ (TNF- $\alpha$ ) in COPD, induces an immune suppressive effect within the tumor microenvironment thereby promoting lung tumorigenesis.

By understanding the common signaling pathways and the key factors helps to develop treatment not only for COPD patients but also to reduce the risk of developing lung cancer in these patients. 
Though several associations between both of these diseases have been elucidated, therapeutic approaches for prevention of lung cancer in COPD patient is still limited. There are new chemical and biological molecules that are effective in animal models but there are no large numbers of multicentre studies. To understand the complexity of these two diseases, studies should involve smokers with premalignant bronchial lesions compared to smokers with and without COPD. Study design should include younger healthy smokers in combination with risk models of lung cancer and COPD which will aid in the identification of new molecular targets in early carcinogenesis and thus leads to the development of early diagnostic tests and novel therapies.

\section{Author's Contributions}

Ms. Geetha Shanmugam has done data collection and wrote the manuscript; Dr. Koustav Sarkar designed the review article and also wrote the manuscript; Ms. Sudeshna Rakshit has made the figures for the manuscript.

\section{Acknowledgement}

This research was financially supported by Science and Engineering Research Board (SERB), Department of Science and Technology (DST), Govt. of India (Sanction Order No. "ECR/2016/000965”).

\section{References}

1. Mathers CD, Loncar D. Projections of global mortality and burden of disease from 2002 to 2030. PLoS Med. 2006; 3: e442.

2. WHO. COPD predicted to be third leading cause of death in 2030. 2008.

3. Global Strategy for the Diagnosis, Management, and Prevention of Chronic Obstructive Pulmonary Disease-2018 Report. 2018.

4. Forum of International Respiratory Societies. Lung cancer 2020 Fact sheet 2020.

5. Carr LL, Jacobson S, Lynch DA, Foreman MG, Flenaugh EL, Hersh CP, et al. Features of COPD as predictors of lung cancer. Chest. 2018; 153: 13261335

6. Punturieri A, Szabo E, Croxton TL, Shapiro SD, Dubinett SM. Lung cance and chronic obstructive pulmonary disease: needs and opportunities for integrated research. J Natl Cancer Inst. 2009; 101: 554-559.

7. Butler SJ, Ellerton L, Goldstein RS, Brooks D. Prevalence of lung cancer in chronic obstructive pulmonary disease: A systematic review. Respiratory Medicine: X. 2019; 1: 100003

8. Sundar IK, Mullapudi N, Yao H, Spivack SD, Rahman I. Lung cancer and its association with chronic obstructive pulmonary disease: update on nexus of epigenetics. Curr Opin Pulm Med. 2011; 17: 279-285.

9. Houghton AM. Mechanistic links between COPD and lung cancer. Nat Rev Cancer. 2013; 13: 233-245.

10. De Torres JP, Bastarrika G, Wisnivesky JP, Alcaide AB, Campo A, Seijo LM et al. Assessing the relationship between lung cancer risk and emphysema detected on low-dose CT of the chest. Chest. 2007; 132: 1932-1938.

11. Lawrence MS, Stojanov $P$, Polak $P$, et al., Mutational heterogeneity in cance and the search for new cancer-associated genes. Nature. 2013; 499: 214 218.

12. Wu X, Yuan B, Lopez E, Bai C, Wang X. Gene polymorphisms and chronic obstructive pulmonary disease, J Cell Mol Med. 2014; 18: 15-26.

13. Zhou J, Zhu Z, Bai C, Sun H, Wang X. Proteomic profiling of lymphocytes inautoimmunity, inflammation and cancer, J Transl Med. 2014; $12: 6$.

14. Kim WJ, Oh YM, Kim TH, Lee JiH, Kim EK, Lee JH, et al. CHRNA3 variant for lung cancer is associated with chronic obstructive pulmonary disease in
Korea. Respiration. 2013; 86: 117-122.

15. Yang L, Qiu F, Lu X, Huang D, Ma G, Guo Y, et al. Functional polymorphisms of CHRNA3 predict risksof chronic obstructive pulmonary disease and lung cancer in Chinese. PLoSOne. 2012; 7: e46071.

16. Liu Y, Kheradmand F, Davis CF, et al. Focused analysis of exome sequencingdata for rare germline mutations in familial and sporadic lung cancer, J.Thorac. Oncol. 2016; 11: 52-61.

17. Li Y, Huang J, Amos Cl. Genetic association analysis of complex diseases in corporating intermediate phenotype information. PLoSOne. 2012; 7: e46612.

18. Stankovic MM, Nestorovic AR, Tomovic AM, et al. TNF-alpha-308promotor polymorphism in patients with chronic obstructive pulmonarydisease and lung cancer, Neoplasma. 2009; 56: 348-352.

19. Ziolkowska-Suchanek I, Mosor M, Gabryel P, et al., Susceptibility loci in lungcancer and COPD: association of IREB2 and FAM13A with pulmonary diseases, Sci Rep. 2015; 5: 13502.

20. Yang L, Lu X, Qiu F, et al. Duplicated copy of CHRNA7 increases risk andworsens prognosis of COPD and lung cancer, Eur J Hum Genet. 2015; 23: $1019-1024$

21. Suzuki M, Wada H, Yoshino $M$, et al. Molecular characterization of chronicobstructive pulmonary disease-related non-small cell lung cancer throughaberrant methylation and alterations of EGFR signaling, Ann Surg Oncol. 2010; 17: 878-888.

22. Shaykhiev R, Sackrowitz R, Fukui $T$, et al. Smoking-induced CXCL14expression in the human airway epithelium links chronic obstructive pulmonary disease to lung cancer, Am J Respir Cell Mol Biol. 2013; 49: 418-425.

23. Wang DC, Shi L, Zhu Z, Gao D, Zhang Y. Genomic mechanisms of transformation from chronic obstructive pulmonary disease to lung cancer. Seminars in Cancer Biology. 2017; 42: 52-59.

24. Tessema M, Yingling CM, Picchi MA, Wu G, Liu Y, Weissfeld JL, et al. Epigenetic repression of $\mathrm{CCDC} 37$ and MAP1B links chronicobstructive pulmonary disease to lung cancer. J Thorac Oncol. 2015; 10: 1181-1188.

25. Qiu W, Baccarelli A, Carey VJ, et al. Variable DNA methylation is associated with chronicobstructive pulmonary disease and lung function, Am J Respir Crit Care Med. 2012; 185: 373-381.

26. Suzuki M, Wada $\mathrm{H}$, Yoshino $\mathrm{M}$, et al. Molecular characterization of chronic obstructive pulmonary disease-related nonsmall cell lung cancer through aberrant methylation and alterations of EGFR signaling. Ann Surg Oncol. 2010; 17: 878-888.

27. Wang R, An J, Ji F, et al. Hypermethylation of the Keap1 gene in human lung cancer cell lines and lung cancer tissues. Biochem Biophys Res Commun. 2008; 373: 151-154.

28. Zelko IN, Mueller MR, Folz RJ. CpG methylation attenuates Sp1 and Sp3 binding to the human extracellular superoxide dismutase promoter and regulates its cell-specific expression. Free Radic Biol Med. 2010; 48: 895904.

29. Sood A, Petersen H, Blanchette CM, et al. Wood smoke exposure and gene promoter methylation are associated with increased risk for COPD in smokers. Am J Respir Crit Care Med. 2010; 182: 1098-1104.

30. Lin RK, Hsieh YS, Lin P, et al. The tobacco-specific carcinogen NNK induces DNA methyltransferase 1 accumulation and tumor suppressor gene hypermethylation in mice and lung cancer patients. J Clin Invest. 2010; 120: 521-532.

31. Ruthenburg AJ, Li H, Patel DJ, et al. Multivalent engagement of chromatin modifications by linked binding modules. Nat Rev Mol Cell Biol. 2007; 8: 983-994.

32. Berger SL. The complex language of chromatin regulation during transcription Nature. 2007; 447: 407-412.

33. Guil S, Esteller M. DNA methylomes, histone codes and miRNAs: tying it all together. Int J Biochem Cell Biol. 2009; 41: 87-95. 
34. Jirtle RL, Skinner MK. Environmental epigenomics and disease susceptibility. Nat Rev Genet. 2007; 8: 253-262.

35. Rajendrasozhan S, Yao H, Rahman I. Current perspectives on role of chromatin modifications and deacetylases in lung inflammation of COPD. COPD. 2009; 6: 291-297.

36. Ito K, Ito M, Elliott WM, Cosio B, Caramori G, Kon OM, et al. Decreased histone deacetylase activity in chronic obstructive pulmonary disease. N Engl J Med. 2005; 352: 1967-1976.

37. Ito K, Lim S, Caramori G, Chung KF, Barnes PJ, Adcock IM. Cigarette smoking reduces histone deacetylase 2 expression, enhances cytokine expression, and inhibits glucocorticoid actions in alveolar macrophages. FASEB J. 2001; 15: 1110-1112.

38. Barnes PJ. Role of HDAC2 in the Pathophysiology of COPD. Annu Rev Physiol. 2008; 71: 451-464.

39. Yang SR, Chida AS, Bauter MR, Shafiq N, Seweryniak K, Maggirwar SB, et al. Cigarette smoke induces proinflammatory cytokine release by activation of NF-kappaB and posttranslational modifications of histone deacetylase in macrophages. Am J Physiol Lung Cell Mol Physiol. 2006; 291: L46-57.

40. Marwick JA, Kirkham PA, Stevenson CS, Danahay H, Giddings J, Butler K, et al. Cigarette smoke alters chromatin remodeling and induces proinflammatory genes in rat lungs. Am J Respir Cell Mol Biol. 2004; 31: 633-642.

41. Cosio BG, Tsaprouni L, Ito K, Jazrawi E, Adcock IM, Barnes PJ. Theophylline restores histone deacetylase activity and steroid responses in COPD macrophages. J Exp Med. 2004; 200: 689-695.

42. Tsai SC, Seto E. Regulation of histone deacetylase 2 by protein kinase CK2 J Biol Chem. 2002; 277: 31826-31833.

43. Adenuga D, Yao H, March TH, Seagrave J, Rahman I. Histone deacetylase 2 is phosphorylated, ubiquitinated and degraded by cigarette smoke. Am J Respir Cell Mol Biol. 2008; 40: 464-473.

44. Rajendrasozhan S, Yang SR, Edirisinghe I, Yao H, Adenuga D, Rahman I. Deacetylases and NFkappaB in redox regulation of cigarette smoke-induced lung inflammation: epigenetics in pathogenesis of COPD. Antioxid Redox Signal. 2008; 10: 799-811.

45. Marwick JA, Kirkham PA, Stevenson CS, Danahay H, Giddings J, Butler K, et al. Cigarette smoke alters chromatin remodeling and induces proinflammatory genes in rat lungs. Am J Respir Cell Mol Biol. 2004; 31: 633-642.

46. Rajendrasozhan S, Yang SR, Kinnula VL, Rahman I. SIRT1, an antiinflammatory and antiaging protein, is decreased in lungs of patients with chronic obstructive pulmonary disease. Am J Respir Crit Care Med. 2008; 177: 861-870.

47. Yang SR, Wright J, Bauter M, Seweryniak K, Kode A, Rahman I. Sirtuin regulates cigarette smokeinduced proinflammatory mediator release via RelA/p65 NF-kappaB in macrophages in vitro and in rat lungs in vivo: implications for chronic inflammation and aging. Am J Physiol Lung Cell Mol Physiol. 2007; 292: L567-576.

48. Ito K, Hanazawa T, Tomita K, Barnes PJ, Adcock IM. Oxidative stress reduces histone deacetylase 2 activity and enhances IL-8 gene expression: role of tyrosine nitration. Biochem Biophys Res Commun. 2004; 315: 240-245.

49. Cosio BG, Tsaprouni L, Ito K, Jazrawi E, Adcock IM, Barnes PJ. Theophylline restores histone deacetylase activity and steroid responses in COPD macrophages. J Exp Med. 2004; 200: 689-695.

50. Nott A, Watson PM, Robinson JD, Crepaldi L, Riccio A. S-Nitrosylation of histone deacetylase 2 induces chromatin remodelling in neurons. Nature. 2008; 455: 411-415

51. Meja KK, Rajendrasozhan S, Adenuga D, Biswas SK, Sundar IK, Spooner $\mathrm{G}$, et al. Curcumin restores corticosteroid function in monocytes exposed to oxidants by maintaining HDAC2. Am J Respir Cell Mol Biol. 2008; 39: 312323.

52. Kouzarides T. Chromatin modifications and their function. Cell. 2007; 128 693-705.

53. Tian X, Fang J. Current perspectives on histone demethylases. Acta Biochim
Biophys Sin (Shanghai). 2007; 39: 81-88.

54. Yang J, Jubb AM, Pike L, et al. The histone demethylase JMJD2B is regulated by estrogen receptor alpha and hypoxia, and is a key mediator of estrogen induced growth. Cancer Res. 2010; 70: 6456-6466.

55. Krieg AJ, Rankin EB, Chan D, et al. Regulation of the histone demethylase JMJD1A by hypoxiainducible factor 1 alpha enhances hypoxic gene expression and tumor growth. Mol Cell Biol. 2010; 30: 344-353.

56. Zhou $X$, Sun $\mathrm{H}$, Chen $\mathrm{H}$, et al. Hypoxia induces trimethylated $\mathrm{H} 3$ lysine 4 by inhibition of JARID1A demethylase. Cancer Res. 2010; 70: 4214-4221.

57. Jiang G, Liu CT, Zhang WD, et al. IL-17A and GDF15 are able to induce epithelial-mesenchymal transition of lung epithelial cells in response to cigarette smoke. Exp Ther Med. 2018; 16: 12-20.

58. Gong L, Da Silva Caetano M, Cumpian AM, et al. Tumor necrosis factor links chronic obstructive pulmonary disease and K-ras mutant lung cancer through induction of an immunosuppressive pro-tumor microenvironment. Oncoimmunol. 2016; 5: e1229724.

59. Wiegman $\mathrm{CH}$, Michaeloudes $\mathrm{C}$, Haji G, et al. Oxidative stress-induced mitochondrial dysfunction drives inflammation and airway smooth muscle remodeling in patients with chronic obstructive pulmonary disease. J Allergy Clin Immunol. 2015; 136: 769-780.

60. Pierrou S, Broberg P, O'Donnell RA, et al. Expression of genes involved in oxidative stress responses in airway epithelial cells of smokers with chronic obstructive pulmonary disease. Am J Respir Crit Care Med. 2007; 175: 577 586.

61. Yamada K, Asai K, Nagayasu F, et al. Impaired nuclear factor erythroid 2-related factor 2 expression increases apoptosis of airway epithelial cells in patients with chronic obstructive pulmonary disease due to cigarette smoking. BMC Pulm Med. 2016; 16: 27

62. Pastor MD, Nogal A, Molina-Pinelo S, et al. Identification of oxidative stress related proteins as biomarkers for lung cancer and chronic obstructive pulmonary disease in bronchoalveolar lavage. Int J Mol Sci. 2013; 14: 3440 3455.

63. Martinon F, Burns K, Tschopp J. The inflammasome: a molecular platform triggering activation of inflammatory caspases and processing of prolL-beta. Mol Cell. 2002; 10: 417-426.

64. Ballweg K, Mutze K, Konigstoff $M$, et al. Cigarette smoke extract affects mitochondrial function in alveolar epithelial cells. Am J Physiol Lung Cell Med Physiol. 2014; 307: L895-L907.

65. Lou Y, Li R, Liu J, et al. Mitofusin-2 over-expresses and leads to dysregulation of cell cycle and cell invasion in lung adenocarcinoma. Med Oncol. 2015; 32: 132.

66. Hogg JC, Chu F, Utokaparch S, Woods R, Elliott WM, Buzatu L, et al. The nature of small-airway obstruction in chronic obstructive pulmonary disease. N Engl J Med. 2004; 350: 2645-2653.

67. Bhat TA, Panzica L, Kalathil SG, Thanavala Y. Immune dysfunction in patients with chronic obstructive pulmonary disease. Ann Am Thorac Soc. 2015; 12: S169-S175.

68. Virk H, Arthur G, Bradding P. Mast cells and their activation in lung disease. Transl Res. 2016; 174: 60-76

69. Rijavec M, Volarevic S, Osolnik K, Kosnik M, Korosec P. Natural killer T cells in pulmonary disorders. Respir Med. 2011; 105: S20-S25.

70. Di Stefano A, Caramori G, Gnemmi I, et al. T helper type 17-related cytokine expression is increased in the bronchial mucosa of stable chronic obstructive pulmonary disease patients. Clin Exp Immunol. 2009; 57: 316-324.

71. Hodge S, Tran HB, Hamon R, et al. Nonantibiotic macrolides restore airway macrophage phagocytic function with potential anti-inflammatory effects in chronic lung diseases. Am J Physiol Lung Cell Mol Physiol. 2017; 312: L678L687.

72. Sekine $\mathrm{Y}$, Hata A, Koh E, Hiroshima K. Lung carcinogenesis from chronic obstructive pulmonary disease: characteristics of lung cancer from COPD and contribution of signal transducers and lung stem cells in the inflammatory 
microenvironment. Gen Thorac Cardiovasc Surg. 2014; 62: 415-421.

73. Greten FR, Karin M. The IKK/NF-kappaB activation pathway-a target for prevention and treatment of cancer. Cancer Lett. 2004; 206: 193-199.

74. Park S, Zhang X, Li C, Yin C, Li J, Fallon JT, et al. Single-cell RNA sequencing reveals an altered gene expression pattern as a result of CRISPR/cas9mediated deletion of Gene 33/Mig6 and chronic exposure to hexavalent chromium in human lung epithelial cells. Toxicol Appl Pharm. 2017; 330: 30-39.

75. Manguso RT, Pope HW, Zimmer MD, et al. In vivo CRISPR screening identifies Ptpn2 as a cancer immunotherapy target. Nature. 2017; 547: 413418 .

76. Li L, Li W, Chen N, et al. FLI1 exonic circular RNAs as a novel oncogenic driver to promote tumor metastasis in small cell lung cancer. Clin Cancer Res. 2019; 25: 1302-1317.

77. Wang K, Xing ZH, Jiang QW, et al. Targeting uPAR by CRISPR/Cas9 system attenuates cancer malignancy and multidrug resistance. Front Oncol. 2019; 9: 80 .

78. Lu Y, Xue J, Deng T, et al. Safety and feasibility of CRISPR-edited T cells in patients with refractory non-small-cell lung cancer, Nature Med. 2020; 26 $732-740$.

79. Durham AL, Adcock IM. The relationship between COPD and lung cancer. Lung Cancer. 2017; 90: 121-127.

80. Rusca N, Monticelli S. MiR-146a in immunity and disease. Mol Biol Int. 2011 2011: 437301.

81. Osei ET, Florez-Sampedro L, Tasena $\mathrm{H}$, et al. miR-146a-5p plays an essential role in the aberrant epithelial- fibroblast cross-talk in COPD. Eur Respir J. 2017; 49: 1602538.

82. Conickx G, Mestdagh P, Avila Cobos F, et al. MicroRNA profiling reveals a role for microRNA-218-5p in the pathogenesis of chronic obstructive pulmonary disease. Am J Respir Crit Care Med. 2017; 195: 43-56.
83. Cho WC, Chow AS, Au JS. Restoration of tumour suppressor hsa-miR-145 inhibits cancer cell growth in lung adenocarcinoma patients with epidermal growth factor receptor mutation. Eur J Cancer. 2009; 45: 2197-2206.

84. Liu X, Sempere LF, Ouyang $\mathrm{H}$, et al. MicroRNA-31 functions as an oncogenic microRNA in mouse and human lung cancer cells by repressing specific tumor suppressors. J Clin Invest. 2010; 120: 1298-1309.

85. Ebi $\mathrm{H}$, Sato $\mathrm{T}$, Sugito $\mathrm{N}$, Hosono $\mathrm{Y}$, Yatabe $\mathrm{Y}$, Matsuyama $\mathrm{Y}$, et al. Counterbalance between RB inactivation and miR-17-92 overexpression in reactive oxygen species and DNA damage induction in lung cancers. Oncogene. 2009; 28: 3371-3379.

86. Guo L, Liu Y, Bai Y, Sun Y, Xiao F, Guo Y. Gene expression profiling of drug-resistant small cell lung cancer cells by combining microRNA and cDNA expression analysis. Eur J Cancer. 2010; 46: 1692-702.

87. Zhu W, Shan X, Wang T, Shu Y, Liu P. MiR-181b modulates multidrug resistance by targeting BCL2 in human cancer cell lines. Int J Cancer. 2010; 127: $2520-2529$

88. Ma L, Reinhardt F, Pan E, et al. Therapeutic silencing of miR-10b inhibits metastasis in a mouse mammary tumor model. Nat Biotechnol. 2010; 28 341-347.

89. Donnelly LE, Barnes PJ. Chemokine receptors as therapeutic targets in chronic obstructive pulmonary disease. Trends in Pharmacol Sci. 2006; 27: 546-553.

90. Dean EJ, Ranson M, Blackhall F, Holt SV, Dive C. Novel therapeutic targets in lung cancer: Inhibitor of apoptosis proteins from laboratory to clinic. Cancer Treat Rev. 2007; 33: 203- 212.

91. Rahman I. Antioxidant therapies in COPD, Int J Chron Obstruct Pulmon Dis. 2006; 1: 15-29.

92. Mercer BA, D'Armiento JM. Emerging role of MAP kinase pathways as therapeutic targets in COPD. Int J COPD. 2006; 1: 137-150. 